1 University of Leeds, Leeds Teaching Hospitals NHS Trust and Leeds Community Healthcare NHS long covid service

2 Centre for Patient Reported Outcome Research, University of Birmingham

3 Faculty of Medicine, Health, and Life Science; Swansea University

4 University of Birmingham Cite this as: $B M J$ 2022;376:0257 http://dx.doi.org/10.1136/bmj.0257 Published: 28 January 2022

\section{Using condition specific patient reported outcome measures for long covid}

\author{
Manoj Sivan, ${ }^{1}$ Shaney Wright, Sarah Hughes, ${ }^{2,3}$ Melanie Calvert ${ }^{4}$
}

Management expert Peter Drucker's famous words "if you can't measure it, you can't manage it" holds good for post-covid syndrome, or long covid, which affects an estimated two million individuals in the $\mathrm{UK}^{1}$ and extrapolated prevalence data suggests more than 50 million individuals may be affected worldwide. ${ }^{2}$ Long covid is a multisystem condition with over 200 symptoms reported across most of the organ systems, often with a chronic fluctuating pattern of presentation. ${ }^{3}$ With up to $30 \%$ of covid- 19 health burden being related to covid induced disability, long covid presents substantial challenges for healthcare systems worldwide. ${ }^{4}$ Management of this complex syndrome, in part, requires new integrated long covid services with expertise drawn from a range of specialists across disciplines from both primary and secondary care. ${ }^{5}$ Healthcare services globally are investing in these new pathways of care, but there are no agreed measurement metrics yet to comprehensively capture patient experience or the effectiveness of treatments, partly due to lack of clear biomarkers for the condition.

Patient reported outcome measures (PROMs) are questionnaire tools to ascertain patients' views of their symptoms, their functional status, and their health-related quality of life. ${ }^{7}$ PROM use in other routine clinical contexts have been shown to facilitate communication, engage patients in their care, tailor care to individual patients' needs, and show value for money for those investing in the services. ${ }^{89}$ Clinical outcome assessments should include clinically important concepts that define the disease in the target population, assess the impact of disease, and reflect the lived experience of those with the condition. Given the large scale, relative novelty, and multifariousness of long covid syndrome, coupled with shortcomings in understanding viral-onset illness, it is unsurprising that standardised assessments of functioning, disability, and health are lacking. ${ }^{10} 11$

Currently long covid services are using PROMs developed for other conditions such respiratory conditions (Medical Research Council Dyspnea Scale), anxiety disorder (Generalised Anxiety Disorder Assessment) and depression (Patient Health Questionnaire) and a range of other symptom-specific PROMs that have not yet been validated for use with long covid. ${ }^{12-14}$ However, this approach has several limitations. Such measures, in our experience, are cognitively burdensome to long covid patients, do not comprehensively capture the spectrum of symptoms, cannot directly engage with the underlying biological mechanisms, and are reported not to be meaningful by patients, families, and clinicians. Using a range of symptom specific measures makes it challenging to repeat the measures frequently to capture day by day fluctuations and are difficult to implement in busy services overburdened with managing such a large caseload of patients. There is the added danger of misleading management, for example individuals scoring highly on anxiety scores may get diverted to psychological services when their anxiety is being driven by underlying dysautonomia (increased heart rate) which needs medical optimisation.

Clinicians, services, and researchers need to invest their energies in developing and validating long covid specific PROMs or validating existing PROMs for use in long covid routine clinical practice and research settings for long covid. Condition specific PROMs can provide valuable information on symptom range, severity, functional impact-and more importantly-in combination with biomedical research, help understand underlying mechanisms, phenotypes, and traits in this heterogeneous clinical syndrome. ${ }^{11}$ Such measures can be used to support individuals with self-management and monitoring, in addition to supporting healthcare services to align long covid care with health system goals. The PROMs need to measure not only symptom range, but also burden in daily activities, including impact on family life, leisure, and work. The World Health Organisation (WHO) provides a very useful framework of and Health (ICF) to understand the various aspects of any health condition and its interaction with the affected individual which could be conceptualised in selecting measures to understand long covid in its totality. ${ }^{15}$

Patient groups-including under-served populations-need to be involved in the development, selection, and co-design of systems to implement long covid specific PROMs in care pathways. ${ }^{8}$ There needs to be early engagement with other key stakeholders-clinicians, health informatics, governance, researchers, and service commissioners. When using a combination of measures, we need to develop a least burdensome set of PROMs that can be used across clinical and research settings and applicable to low, middle-, and high-income countries. PROMs should empower patients to self-monitor their condition and help them understand the physical, cognitive, and emotional triggers of their condition. ${ }^{16}$ Healthcare services should also be able to use these PROMs to evaluate their investment and effectiveness of the care being delivered. Through careful selection and robust, well planned implementation, PROMs, as part of a combination of initiatives, have potential to enhance the care of the millions of people living with long covid. International Classification of Functioning Disability 


\section{OPINION}

Competing interests: MS/SW have led the development of the COVID-19 Yorkshire Rehabilitation Scale (C19-YRS). SH/MC have led the development of the Symptom Burden Questionnaire-Long COVID. MC is NIHR senior investigator, Director Centre for Patient-Reported Outcomes Research, Director Birmingham Health Partners Centre for Regulatory Science and Innovation, and co-investigator NIHR Biomedical Research Centre Birmingham, NIHR Applied Research Collaboration West Midlands and HDRUK.

The views expressed in this article are those of the author(s) and not necessarily those of the NIHR, UKRI or Department of Health and Social Care.

1 Whitaker M. J. E, Chaadeau-Hyam M. Persistent symptoms following SARS-CoV-2 infection in a random community sample of 508707 people.medRxiv . 2021.

2 Hirschtick JL, Titus AR, Slocum E, etal. Population-Based Estimates of Post-acute Sequelae of Severe Acute Respiratory Syndrome Coronavirus 2 (SARS-CoV-2) Infection (PASC) Prevalence and Characteristics. Clin Infect Dis2021;73:2055-64. doi: 10.1093/cid/ciab408 pmid: 34007978

3 Brown DA, O'Brien KK. Conceptualising Long COVID as an episodic health condition. BMJ Glob Health 2021;6:e007004. doi: 10.1136/bmigh-2021-007004 pmid: 34551971

4 Briggs A, Vassall A. Count the cost of disability caused by COVID-19. Nature 2021;593:502-5. doi: 10.1038/d41586-021-01392-2 pmid: 34040204

5 NHSEngland. National guidance for post-COVID syndrome assessment clinics. 2021.

6 Sivan M, Rayner C, Delaney B. Fresh evidence of the scale and scope of long covid. BMJ 2021;373:n853. doi: 10.1136/bmj.n853 pmid: 33795224

7 Black N. Patient reported outcome measures could help transform healthcare. BM/2013;346:167. doi: 10.1136/bmj.f167 pmid: 23358487

8 Snyder CF, Aaronson NK, Choucair AK, etal. Implementing patient-reported outcomes assessment in clinical practice: a review of the options and considerations. Qual Life Res 2012;21:1305-14. doi: 10.1007/s11136-011-0054-x pmid: 22048932

9 Calvert M, Kyte D, Price G, Valderas JM, Hjollund NH. Maximising the impact of patient reported outcome assessment for patients and society. BMJ2019;364:k5267. doi: 10.1136/bmj.k5267 pmid: 30679170

10 O'Connor RJ, Preston N, Parkin A, etal. The COVID-19 Yorkshire Rehabilitation Scale (C19-YRS): Application and psychometric analysis in a post-COVID-19 syndrome cohort. J Med Virol 2021.pmid: 34676578

11 Sivan M, Parkin A, Makower S, Greenwood DC. Post-COVID syndrome symptoms, functional disability, and clinical severity phenotypes in hospitalized and nonhospitalized individuals: A cross-sectional evaluation from a community COVID rehabilitation service. J Med Virol 2021. doi: $10.1002 /$ /mv. 27456 pmid: 34783052

12 Heightman M, Prashar J, Hillman TE, etal. Post-COVID-19 assessment in a specialist clinical service: a 12-month, single-centre, prospective study in 1325 individuals. BMJ Open Respir Res 2021;8:e001041. doi: 10.1136/bmiresp-2021-001041 pmid: 34764200

13 Evans RA, McAuley H, Harrison EM, etalPHOSP-COVID Collaborative Group. Physical, cognitive, and mental health impacts of COVID-19 after hospitalisation (PHOSP-COVID): a UK multicentre, prospective cohort study. Lancet Respir Med 2021;9:1275-87. doi: 10.1016/S2213-2600(21)00383-0 pmid: 34627560

14 Parkin A, Davison J, Tarrant R, etal. A Multidisciplinary NHS COVID-19 Service to Manage Post-COVID-19 Syndrome in the Community. J Prim Care Community Health 2021;12:21501327211010994. doi: 10.1177/21501327211010994 pmid: 33880955

15 Patel K, Straudi S, Yee Sien N, Fayed N, Melvin JL, Sivan M. Applying the WHO ICF Framework to the Outcome Measures Used in the Evaluation of Long-Term Clinical Outcomes in Coronavirus Outbreaks. Int J Environ Res Public Health 2020;17:E6476. doi: 10.3390/ijerph17186476 pmid: 32899534

16 WHO. Support for rehabilitation: self-management after COVID-19-related illness. Copenhagen: WHO Regional Office for Europe; 2021. Available from: https://apps.who.int/iris/handle/10665/344472. 